\title{
La prosodie comme ressource pour l'organisation de l'interaction : état des lieux et illustrations
}

\author{
Rasmus Persson, Université de Linköping \& Université d'York
}

\begin{abstract}
Résumé : Cet article offre un état des lieux de la recherche portant sur les liens entre la prosodie et l'organisation de l'interaction sociale. Nous faisons un survol des principaux travaux sur le formatage prosodique et phonétique et sa pertinence procédurale pour l'interaction, selon trois axes : la gestion de la parole, l'organisation séquentielle, et la construction des actions. Dans chaque cas, nous offrons également des analyses illustratives de données en français.
\end{abstract}

\begin{abstract}
This article takes stock of the current state of research on the connections between prosody and the organisation of social interaction. An overview is given of central studies of prosodic and phonetic design and its procedural relevance for interaction, along three lines of inquiry: the management of turns, sequence organisation, and action formation. For each of these issues, illustrative analyses based on French data are also presented.
\end{abstract}

Mots-clés : interaction sociale, analyse conversationnelle, prosodie, intonation Keywords: social interaction, conversation analysis, prosody, intonation

\section{Introduction}

Si la prosodie de la parole en général est depuis longtemps un objet de recherche dans divers courants linguistiques, l'étude de la prosodie spécifiquement en interaction située est plus récente et relativement moins développée. Néanmoins, les représentants de l'analyse conversationnelle (AC ci-après) sont en général prompts à reconnaître la pertinence potentielle des détails prosodiques (Schegloff 1998). En effet, l'AC avance l'idée qu'il y a de l'ordre en tout point (Sacks 1984, 22) et que tout détail du comportement est a priori potentiellement pertinent (Heritage 1989, 22). Qui plus est, le fait qu'il n'y a pas de parole sans prosodie veut dire que l'analyse auditive de la parole en interaction ne peut en effet s'empêcher de tenir compte de la prosodie, que ce soit explicitement ou non (Walker 2013).

Cette contribution se propose de présenter un aperçu, par nécessité sélectif, des recherches relatives aux façons dont le formatage prosodique de la parole a une incidence sur l'organisation de l'interaction, et vice versa. Ce faisant, nous illustrerons empiriquement quelques-uns des enjeux dans ce champ d'étude, en nous appuyant sur des données en français ${ }^{1}$.

\footnotetext{
${ }^{1}$ Alors que certains extraits présentés dans cet article représentent des collections établies pour des études publiées ou en cours de publication (extraits 1-4 et 8), d'autres extraits doivent plutôt être compris comme étant des expansions inédites de collections préalables, ou comme illustrant des ébauches d'analyses (extraits 5-7). Les extraits sont tirés de diverses interactions, familières et institutionnelles, enregistrées dans leur contexte naturel d'occurrence ; sont représentés des appels téléphoniques (extraits 1-3 et 7-8) et
} 
Pour ce faire, nous nous pencherons successivement sur quelques tâches fondamentales dans la parole en interaction, pour lesquelles la variabilité prosodique sert de ressource aux participants. Premièrement, nous analyserons la prosodie comme ressource pour l'alternance de la parole, ou plus généralement pour la tâche de gérer la distribution de la parole entre les participants. Nous recenserons des études qui démontrent une pertinence procédurale de différentes formes prosodiques pour l'organisation de la parole en TCUs (turn-constructional units) et tours de parole reconnaissables. Deuxièmement, nous discuterons le rôle que la prosodie peut jouer pour l'organisation des séquences d'actions, y compris pour le marquage ou la projection de relations spécifiques entre tours de parole individuels, adjacents ou non. Troisièmement, nous signalerons quelques cas où le format prosodique est essentiel pour la constitution même de l'action accomplie dans et par le tour de parole, c'est-à-dire pour ce que le locuteur fait par le biais du tour de parole, et pour le type de réponse attendue.

Même si certaines notions ayant leur origine dans l'AC ont été adoptées dans d'autres courants linguistiques avec d'autres démarches méthodologiques (par exemple en psycholinguistique (Bögels \& Torreira 2015)), notre revue se limitera essentiellement aux travaux menés dans une perspective conversationnaliste d'inspiration ethnométhodologique. Accordant une place centrale à l'analyse séquentielle, ces recherches s'inscrivent dans l'esprit méthodologique de l'AC et s'alignent avec son objectif de mettre à jour les catégories des participants et les procédés qui en permettent la production. Les principes méthodologiques de ce courant ne seront pas abordés dans cet article (qui se focalise plutôt sur les résultats), mais ils ont été détaillés ailleurs (Local \& Walker 2005 ; Walker 2013).

\section{Prosodie et gestion de la parole}

\subsection{La constitution des TCUs}

La prosodie peut être comprise comme constituée des trois paramètres perceptifs : (1) la mélodie (ou hauteur mélodique, en anglais pitch), (2) la longueur et (3) la sonie (en anglais loudness) ; les principaux corrélats acoustiques de ces trois paramètre sont, respectivement, (1) la fréquence fondamentale, (2) la durée et (3) l'intensité. Dans les recherches sur la prosodie dans l'interaction, la thématique du rôle de la mélodie pour l'alternance de la parole a occupé une place importante, et d'importants résultats en ont émergé. Avant de les détailler, il convient cependant de préciser que la gestion de la parole n'est pas un système mécanique de règles et d'unités au sens strict. Plutôt, les représentants de la linguistique interactionnelle (Ford \& al. 1996, 2013 ; Ford 2004 ; Mondada 2007) appréhendent le TCU comme une unité que les participants projettent de façon dynamique et révisable, et qui se développe au fil de la production de la parole, de manière provisoire et contingente. Plutôt qu'une unité formelle fixe que les participants ne font que réutiliser, le TCU peut être considéré comme un épiphénomène de différentes pratiques pour gérer l'espace alloué pour le tour de parole. Ainsi, il n'est pas possible de détailler exhaustivement les propriétés linguistiques du TCU, et le formatage prosodique n'est pas une exception : il n'y a pas de correspondance simple entre prosodie et complétude du tour. Par exemple, il n'y a pas de contour intonatif spécifique qui projette, en soi, un TRP (un 'point de transition potentiel') à suivre immédiatement. La réserve qui s'impose est donc la suivante : si certaines propriétés prosodiques sont plus fréquentes que d'autres dans les fins des tours de parole, il est généralement reconnu que la gestion de la parole reste un jeu complexe entre la prosodie, la grammaire, la multimodalité, l'action et la séquentialité (Ford \& al. 1996, 2013 ; Mondada 2007). 
Les premiers travaux conversationnalistes sur la prosodie dans la gestion de la parole prenaient pour objet différentes formes de l'anglais (Local \& al. 1985, 1986), et l'anglais n'a cessé d'attirer de l'intérêt dans les recherches en la matière (Wells \& Peppé 1996 ; Wells \& MacFarlane 1998 ; Fox 2001 ; Szczepek Reed 2004, 2010 ; Barth-Weingarten 2016). Un résultat important de ces études sur l'anglais est que les accents majeurs jouent un rôle pour la projection des TRP. La constitution de ces accents majeurs est variable selon la variété régionale, mais ils impliquent une proéminence phonétique manifestée par un mouvement mélodique important et par une sonie (c'est-à-dire l'intensité perçue) et une durée accrues (Wells \& MacFarlane 1998). Le rôle de l'accent majeur est souligné par le fait que le TRP peut commencer avant que le locuteur en exercice n'ait cessé de parler, durant les syllabes inaccentuées qui suivent l'accent majeur, sans que cela pose de problème (Wells \& MacFarlane 1998).

Si l'accentuation primaire du français n'est pas lexicalement variable comme en anglais, mais toujours finale (c'est-à-dire associée à la dernière syllabe pleine des mots ou des groupes de mots (Di Cristo 1998)), nous fournissons ci-dessous quelques indications qu'en français aussi, une place de premier plan est occupée par les accents majeurs (ou nucléaires, dans le sens où les contours intonatifs sont en grande partie déterminés par le mouvement mélodique de la syllabe à accent majeur). D'abord, l'étude des formulations (c'est-à-dire des tours dans lesquels on reformule le dire d'autrui (Heritage \& Watson 1979)) en français a permis de noter provisoirement quelques liens entre la prosodie et l'alternance de la parole (Persson 2013 ; 2014, 65-69). La plus importante proéminence prosodique du TCU en français, qui peut ainsi être identifiée comme l'accent majeur, est généralement portée par la syllabe finale d'un groupe accentuel (bien entendu, il y a aussi des accents primaires qui terminent un groupe accentuel mais non le TCU entier), et manifestée par une durée syllabique accrue et par un mouvement mélodique important (Delais-Roussarie \& al. (2015) sur l'accentuation nucléaire). Typiquement, les tours de formulation s'achèvent après la syllabe qui porte cet accent majeur - et cela vaut pour les deux contours intonatifs attestés : montée finale et montée sur la pénultième (Persson 2013, 2014). La complétude prosodique atteinte après l'accent majeur coïncide en général aussi avec la complétude sur les plans de la lexicosyntaxe et de l'action (Persson 2013). Bien des fois, les transitions entre la formulation et sa réponse se font, après cet accent majeur, sans longs silences ou chevauchements (Persson 2013, 2014) ; voir les lignes 4-6 de l'extrait (1) pour un cas avec montée sur la pénultième. Cet extrait est tiré d'un appel à une université, où l'étudiant CLI cherche à organiser sa réinscription. Comme l'illustre la figure 1, la montée frappe la pénultième -dez de la formulation, et l'accent majeur (bas) tombe sur l'ultime vous.

Extrait (1) ${ }^{2} \quad$ [UBS.421.0:15]

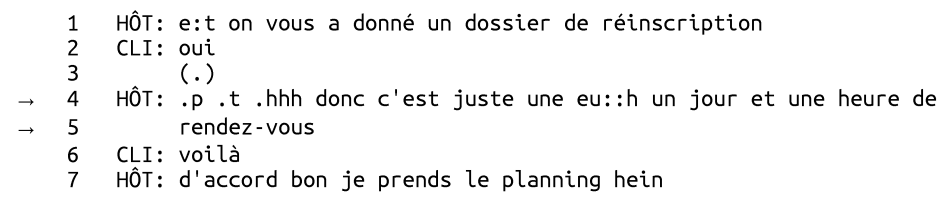

\footnotetext{
${ }^{2}$ Les conventions de transcription sont basées sur le système conventionnel jeffersonien, cf. Persson (2015b) pour une liste précise des conventions employées ici. Les figures montrent, du haut en bas, l'axe temporel, l'oscillogramme, la fréquence fondamentale $\left(f_{0}\right)$ et le matériau verbal découpé en syllabes. Le tracé de $f_{0}$ est présenté sur une échelle logarithmique, et le degré de noirceur représente l'intensité globale (plus noir signifie intensité plus forte (Walker 2012)).
} 


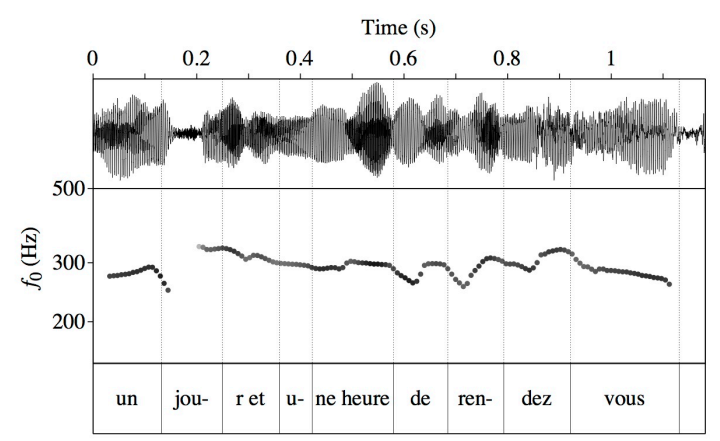

Figure 1. Oscillogramme et tracé de $f_{0}$ du fragment un jour et une heure de rendez-vous (extrait 1).

Or certaines formulations s'étendent après l'accent majeur, avec l'ajout d'une suite de syllabes inaccentuées (un appendice). Dans ces cas-là, la réponse commence parfois en chevauchement, directement après l'accent majeur; cf. l'extrait (2) et la figure 2.

Extrait (2) [CORAL.FTELPV13.1:00]

$\rightarrow \quad 1$ AGE: le début c'est comme Cuba [en f] ait
2 APP: $[\mathrm{mm}]$

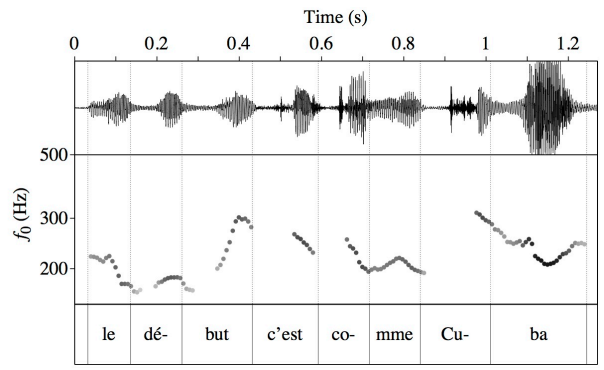

Figure 2. Oscillogramme et tracé de $f_{0}$ du fragment le début c'est comme Cuba (extrait 2).

Dans l'extrait (2), où l'enjeu pour les participants est l'orthographe du nom espagnol Cuba Libre, la pénultième $\mathrm{Cu}$ - porte la montée, et l'accent majeur (bas) de la formulation frappe la syllabe $-b a$ (cf. figure 2 ). La confirmation $(\mathrm{mm})$ arrive immédiatement après cette syllabe, et finit donc en chevauchement avec les syllabes inaccentuées en fait qui sont ajoutées après l'accent majeur. De tels cas révèlent que les participants s'orientent vers la syllabe à accent majeur comme la dernière du TCU; le TRP commence régulièrement après cette syllabe (Persson 2013, 2014). En revanche, les confirmations ne sont typiquement pas amorcées avant la fin de l'accent majeur (mais voir Persson (2014, 68-69) pour l'analyse d'un cas déviant). Que le contour intonatif soit monté sur la pénultième (comme ici) ou en montée finale, l'accent majeur peut donc projeter la fin imminente du TCU.

Une autre indication révélatrice est fournie par les questions lacunaires (Persson 2017), c'est-à-dire les questions formatées comme des énoncés inachevés qui sollicitent leur propre achèvement. Les réponses à ces questions sont ainsi fournies sous la forme de complétions qui 'remplissent le vide'; cf. l'extrait (3) ci-dessous. 
Extrait (3) [CORAL.FTELPV25.1:21]
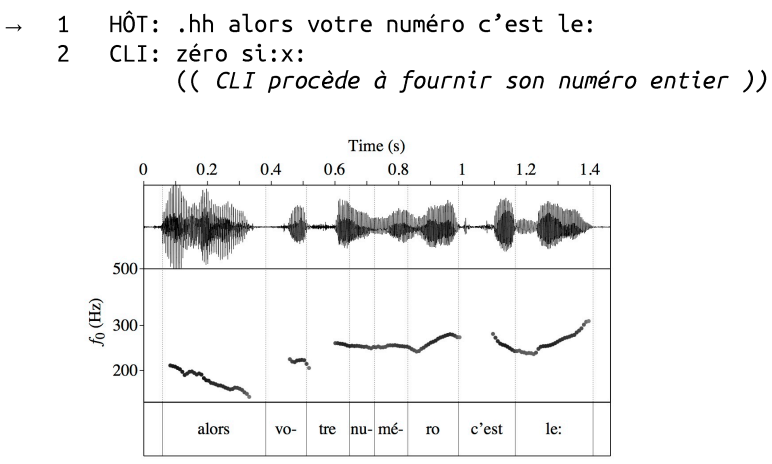

Figure 3. Oscillogramme et tracé de $f_{0} d u$ tour alors votre numéro c'est le (extrait 3).

Dans cette question (cf. fig. 3), la syllabe le: porte l'accentuation majeure qui signale la complétude du tour en dépit de l'incomplétude syntaxique (et cette incomplétude syntaxique sert plutôt à restreindre et le contenu et la forme de la réponse projetée, censée compléter la structure syntaxique). Par ailleurs, on peut noter qu'un contour intonatif spécifique - qui peut être annoté L* $\mathrm{H} \%$ en notation ToBI conventionnelle (Delais-Roussarie \& al. 2015) - est associé à la plupart des questions lacunaires (mais cf. Persson (2017) pour une exception). Or ce contour peut prendre différentes formes selon le phrasé et le matériau verbal. Par exemple, dans la figure 3, il y a notamment une montée tard dans la syllabe à accent majeur (la voyelle de le: commence environ à la hauteur mélodique où se termine la voyelle de c'est). Par contre, dans la question lacunaire dans l'extrait (4), comme il y a un accent sur rue, délimitant le syntagme $c$ 'est vers la rue, la syllabe à accent majeur (de:) manifeste plutôt une descentemontée (la syllabe de: descend, par rapport à rue, avant de monter ; voir fig. 4).

Extrait (4) [OTG.1SB0253.0:14]
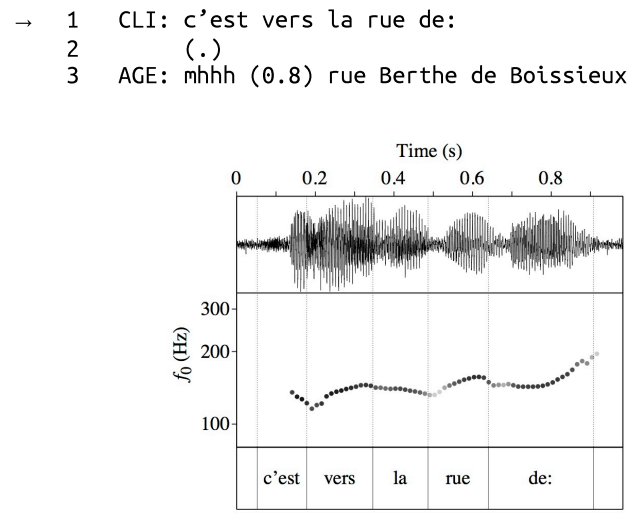

Figure 4. Oscillogramme et tracé de $f_{0} d u$ tour c'est vers la rue de (extrait 4). 
Cette variabilité montre que la forme précise de la courbe intonative peut être reliée au rôle interactionnel de l'intonation, certes, mais la structure métrique (phrastique) du tour ne peut être ignorée non plus. Plus généralement, nous constatons d'une part qu'ici encore, c'est après la syllabe à accent majeur que débute le TRP, et d'autre part que l'accentuation majeure peut servir à accomplir une sorte de complétude prosodique indépendamment de la complétude syntaxique.

Dans la lignée de Szczepek Reed (2004) pour l'anglais, nous notons pour le français que les accents majeurs, et les contours intonatifs qu'ils portent, contribuent à la tâche d'empaqueter les énoncés (et de spécifier leur fonction interactionnelle, cf. section 4 infra). Or la variabilité des contours est telle qu'il n'y a pas lieu de parler d'un contour intonatif 'final', ni 'continuatif' (Walker 2017). Il est intéressant de noter que ces constats cadrent avec les conclusions d'une étude phonétique de corpus sur le français (Torreira \& Valtersson 2015), selon laquelle un seul et même contour intonatif montant est utilisé dans les interrogations totales (qui déboucheraient sur un TRP) et les phrases déclaratives 'continuatives' (qui ne déboucheraient pas sur un TRP).

\subsection{Autres tâches dans la gestion de la parole}

Pour l'anglais, il a été démontré qu'à part la tâche centrale de délimiter le TCU, la prosodie contribue entre autres choses à l'organisation de l'alternance de la parole dans les situations de chevauchement, en distinguant les prises de parole compétitives (manifestant une augmentation globale de la hauteur mélodique et de la sonie) des prises de parole noncompétitives (French \& Local 1983). A été recensé également le rôle significatif des aspects prosodiques dans les pratiques d'expansion du tour au-delà des TRPs effectifs, par exemple les ajouts incrémentaux (Walker 2004) et les raccords abrupts (Local \& Walker 2004). En outre, il y a des pratiques (i.e. les rush-through, définis en grande partie par leur formatage prosodique et phonétique) pour prospectivement neutraliser des TRPs imminents en vue de garder la parole (Walker 2010).

Notamment pour ce qui est de l'allemand, il y a aussi des études qui soulignent l'importance de la prosodie pour constituer des paquets plus grands dans l'interaction, tels les récits (Selting 2000) ou les énumérations (Selting 2007). S'il manque des études analogues pour le français, certaines études dans la tradition phonétique (Portes \& al. 2007) notent que l'intonation d'énumération en français est caractérisée par l'affectation, à la fin de chaque élément énuméré, d'un accent primaire avec un important allongement et avec une mélodie relativement peu dynamique. La montée dite d'énumération, perceptuellement distincte, peut ainsi être distinguée de la montée finale (moins allongée et plus raide) des accents majeurs en fin de TCU. Ainsi, des caractéristiques prosodiques contribuent à la construction de tours multi-TCU, où les TCUs juxtaposés sont des éléments qui exemplifient ou illustrent quelque chose (extrait (5) et figures 5a-c).

Ayant annoncé qu'elle va recevoir une rémunération substantielle, CÉL évoque ici (comme pour rétablir l'équilibre) plusieurs dépenses qu'elle aura à faire, sous forme de propositions parallèles en $\{j$ 'ai ... $\}$ sans coordonnant (comme par exemple une conjonction). CÉL termine l'énumération avec la généralisation tous les frais divers tu vois comme ça (Jefferson 1990). Chacun des frais spécifiques énumérés manifeste un accent allongé et peu dynamique (fig. 5a-c). 
Extrait (5) [CORAL.FPUBDL12.0:36]
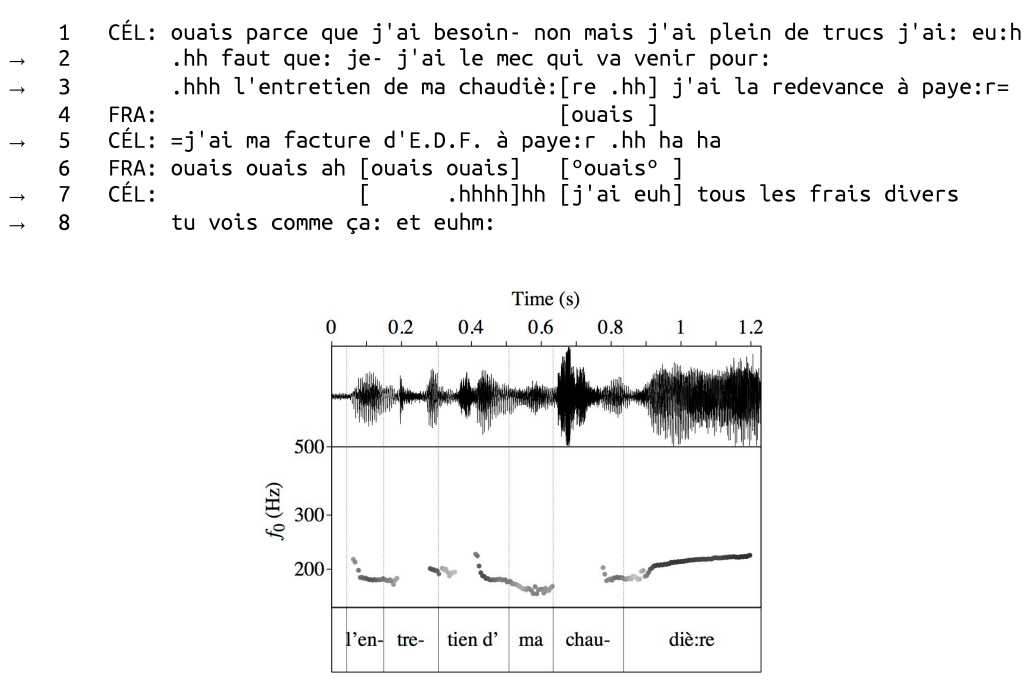

Figure 5a. Oscillogramme et tracé de $f_{0}$ du fragment l'entretien d'ma chaudière (extrait 5).

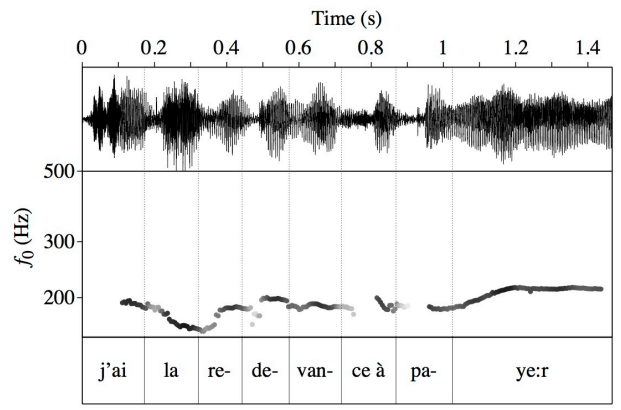

Figure 5b. Oscillogramme et tracé de $f_{0} d u T C U$ j'ai la redevance à payer (extrait 5).

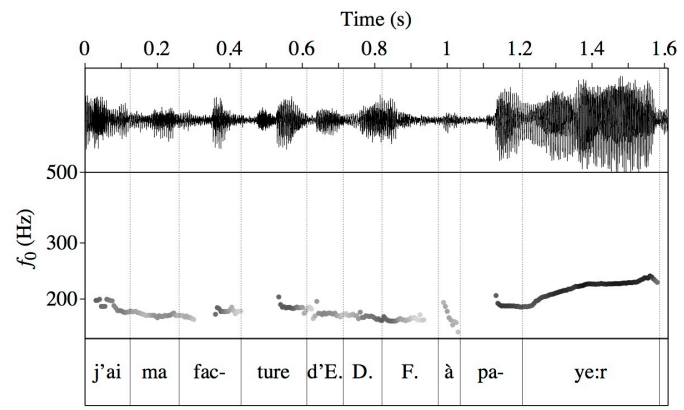

Figure 5c. Oscillogramme et tracé de $f_{0} d u T C U$ j'ai ma facture d'EDF à payer (extrait 5). 
De manière intéressante, ces caractéristiques prosodiques peuvent également être combinées avec des premières parties de paire, telles que les questions totales. Il peut donc s'agir d'un procédé pour construire des questions multi-TCU (multi-alternatives), qui exemplifient plusieurs éléments confirmables/acceptables, tous possibles mais non exhaustifs, plutôt qu'avancer un seul confirmable qui serait ainsi représenté comme le plus pertinent. Nous donnons en exemple l'extrait (6), où JUL accomplit une offre avec une montée 'ordinaire' (ligne 4), pour ensuite refaire son offre avec une montée d'énumération comme le premier élément d'un tour multi-TCU, proposant plusieurs boissons que les invités pourraient accepter (dont le jus de fruits et la sangria sur la table). Ici, les quatre participants sont sur le point de s'installer pour l'apéritif (CLA est déjà assise).

Extrait (6) [CLAPI.chat.3:11]
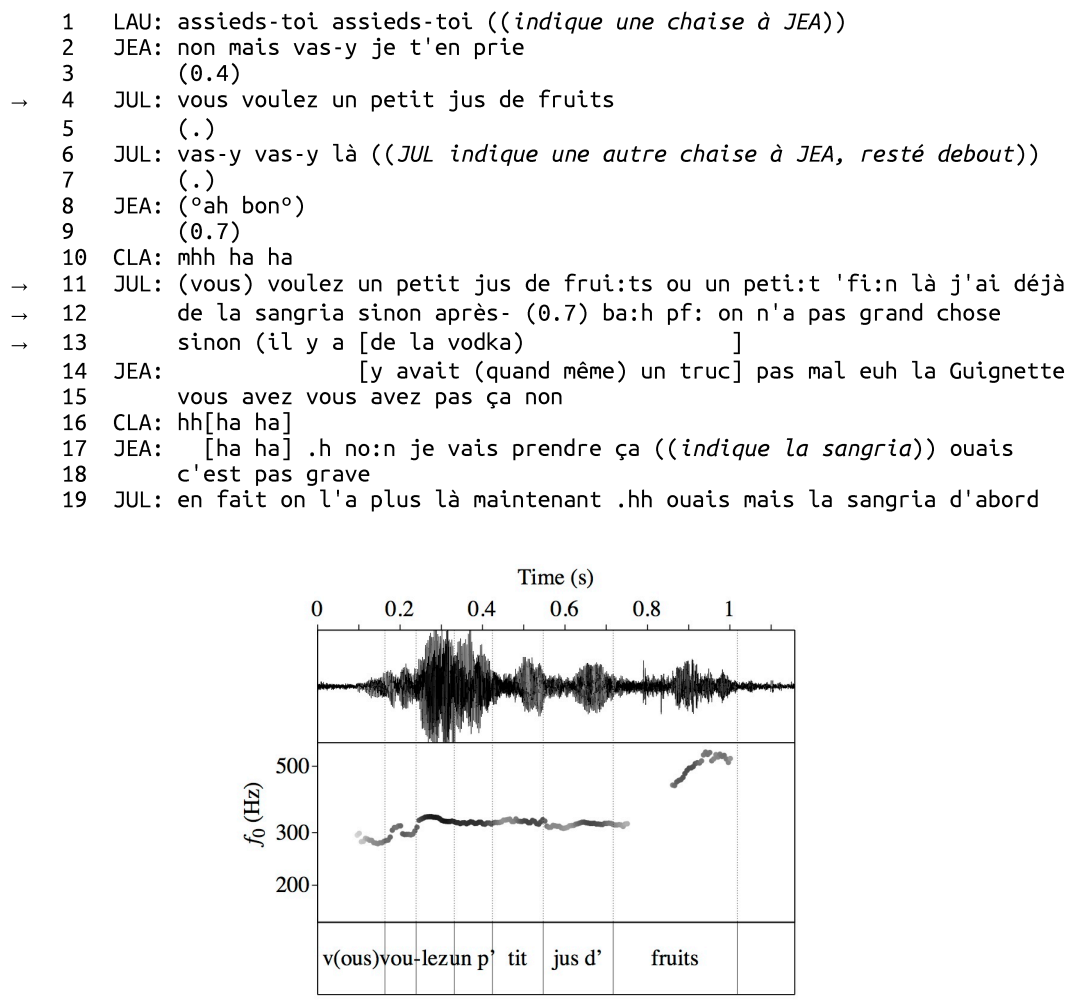

Figure 6a. Oscillogramme et tracé de $f_{0} d u$ tour vous voulez un p'tit jus d'fruits (extrait 6, ligne 4).

Par rapport à la montée de l'offre à la ligne 4 (fig. 6a), la montée d'énumération de la ligne 11 (fig. 6b) est moins raide, vu qu'elle monte moins haut et que la voyelle [i] est allongée (notez aussi que l'amplitude augmente durant la montée). Que l'absence d'une réponse à la première offre soit due à l'activité concurrente de s'installer (voir lignes 6-10) ou au retardement normatif des réponses non préférentielles (ou aux deux), l'offre refaite avec montée d'énumération sert de toute manière à projeter une énumération d'autres alternatives offertes. En effet, le tour de cette offre est continué (ou un peti:t ...) et la boisson nommée la première 
est ainsi 'rétrogradée' en une exemplification des possibilités, parmi d'autres alternatives projetées et ensuite nommées.

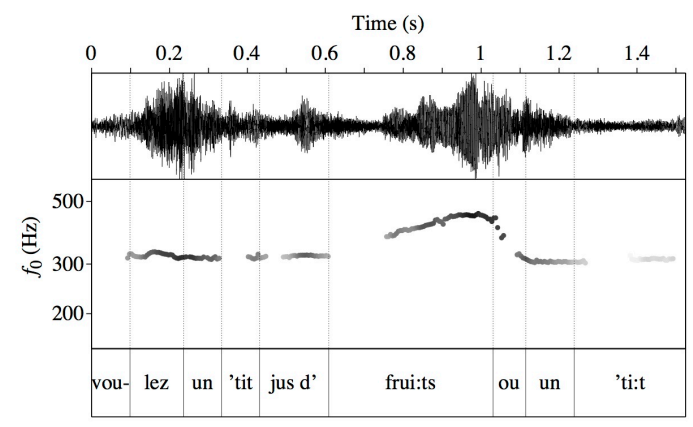

Figure 6b. Oscillogramme et tracé de $f_{0}$ du fragment (vous) voulez un 'tit jus d'fruits ou un 'tit (extrait 6, ligne 11).

Soulignons que ce genre de formatage prosodique ne fonctionne pas de manière indépendante, mais fait partie d'un ensemble plus large de ressources pour formater les confirmables comme étant provisoirement et préliminairement formulés (à côté du simple fait de nommer de multiples alternatives, des ressources syntaxiques comme le simple ou projecteur, et des marques lexicales telles que par exemple). Tout cela vise donc à relâcher l'attente selon laquelle un confirmable nommé soit ‘le bon' (Pomerantz 1988).

Pour en revenir à l'interface entre la prosodie et la tâche générale de gérer la parole, une conclusion préliminaire est la suivante : la prosodie des questions lacunaires et l'intonation dite d'énumération ne sont pas en premier lieu reliées à la délimitation du TCU en soi, mais à la construction de 'paquets' interactionnels de taille variable qui servent à des fins plus spécifiques, ayant trait à l'action. Néanmoins, elles entraînent des répercussions observables sur la manière dont l'alternance de la parole s'organise. La complétude (ou incomplétude) prosodique des tours de parole peut ainsi être comprise comme un sous-produit de la complétude (ou incomplétude) du point de vue de l'action, où la prosodie contribue à l'action plutôt que de réguler directement l'alternance de la parole.

\subsection{Détails phonétiques (non prosodiques)}

Si la prosodie et notamment l'intonation ont attiré une certaine attention dans le cas du français, pour certaines langues, telles que le finnois, le mode phonatoire (c'est-à-dire la qualité de voix) a été démontré comme primordial pour l'alternance de la parole, à la place de l'intonation. Comme le montre Ogden $(2001,2004)$, la pertinence imminente de transition est indiquée vers la fin potentielle du tour au moyen de différentes qualités de voix non modales (voix craquée, voix soufflée/murmurée, chuchotement) et, en outre, l'expiration. En revanche, une occlusion glottale maintenue projette une continuation du tour de parole, en finnois comme en anglais (Local \& Kelly 1986 ; Ogden 2001). Ces résultats ont souligné l'importance d'explorer quelles sont les manifestations sonores de la constitution du tour de parole propres à chaque langue.

En effet, l'étude de l'anglais a révélé la pertinence interactionnelle d'aspects phonétiques qui ne sont pas classés comme prosodiques, mais qui relèvent plutôt de l'articulation et de la phonation (Local \& al. 1986 ; Local \& Kelly 1986 ; Local \& Walker 2012). Ces explorations de phénomènes comme l'anticipation articulatoire et la réalisation variable des occlusives 
servent de rappel que la prosodie n'est pas le seul domaine des formes sonores qui mérite d'être étudié en tant que ressource pour l'interaction généralement, et pour la gestion de la parole en particulier. Un recensement systématique et exhaustif n'a pas encore été fait en ce qui concerne les aspects phonétiques (non prosodiques) du français qui importent à la gestion de la parole. Ici, nous ne pouvons offrir à cet effet que des suggestions et des observations fragmentaires, en esquissant quelques pistes de recherche possibles.

D'abord, mentionnons le cas où la complétude grammaticale et actionnelle coïncide avec un mot qui se termine par une voyelle pleine (c'est-à-dire autre que le $e$ caduc). Des phonéticiens ont observé que des voyelles fermées et mi-fermées peuvent être (partiellement ou totalement) dévoisées et fricativisées en fin d'énoncé, de sorte que par exemple $\{.$. aujourd'hui $\}$ se prononcerait comme [ozusdyiç] et $\{. .$. tout $\}$ s'approcherait de [tux ${ }^{\mathrm{w}}$ (Fagyal \& Moisset 1999), et il semblerait que ce phénomène peut donc fonctionner comme un indice de fin de TCU. Ensuite, dans le cas où la complétude grammaticale et actionnelle coïncide avec un $e$ caduc final, ou avec une consonne finale, il est possible qu'un certain rôle soit joué par réalisation ou non du $e$ caduc, ou du $e$ 'parasitaire', respectivement (Hansen \& Mosegaard Hansen (2003) pour une approche sociolinguistique-pragmatique). Ainsi, certaines voyelles de détente consonantique pourraient éventuellement contribuer au marquage phonétique du TRP. Ces voyelles se distingueraient donc du euh dit d'hésitation (qui projette au contraire une continuation du tour), non seulement en ce qui concerne la durée (plus longue pour ce dernier) mais aussi en ce qui concerne la qualité vocalique (par exemple le euh d'hésitation semble généralement plus arrondi, et souvent plus antérieur). Enfin, parallèlement à l'anglais, où les occlusives sourdes en fin de mot sont relâchées avec aspiration uniquement avant les TRPs (Local \& Walker 2012), il semblerait qu'en français aussi les occlusives sourdes en fin de mot relâchées avec aspiration apparaissent notamment en fin de TCU. Par contre, dans le cas de continuation du tour, les occlusives finales des mots en français sont typiquement relâchées de sorte qu'elles enchaînent directement sur le segment qui suit (sans aspiration ou autre intervalle perceptible entre l'achèvement de l'occlusive et le début du mot suivant), et si ce segment est une voyelle, le phénomène d'enchaînement implique que l'occlusive est même produite comme l'attaque de la syllabe suivante.

Pour conclure, relevons que les propriétés projectives de cette variabilité articulatoire, liée à l'enchaînement des consonnes et voyelles, semblent se situer à une échelle plus locale que les aspects prosodiques, liés surtout à l'agencement des syllabes. Ces deux niveaux de projection ne se substituent pas l'un à l'autre, mais se complètent; s'ils convergent souvent, ils sont néanmoins susceptibles de diverger occasionnellement (Walker 2017).

\section{Prosodie et organisation séquentielle}

Au-delà de la constitution des TCUs, certaines études ont démontré la pertinence de la prosodie et de la phonétique d'une part quant à la tâche d'indiquer à quel tour de parole (précédent ou projeté) se rapporte le TCU actuel, et d'autre part quant à la tâche de spécifier le rapport entre ces tours séquentiellement reliés. Une étude exemplaire est celle de Local (1992) pour l'anglais, décrivant une pratique qui signale qu'un tour redémarré continue une trajectoire séquentielle antérieure, traitant ainsi rétrospectivement le(s) tour(s) intermédiaire(s) comme une digression qui s'éloigne de la trajectoire séquentielle. À savoir, en faisant correspondre la hauteur mélodique et la sonie du tour continuant avec celles du tour continué, le lien séquentiel est établi non avec le(s) tour(s) qui précède(nt) immédiatement, mais avec un tour et une trajectoire séquentielle plus lointains. De manière similaire, Couper-Kuhlen

\footnotetext{
${ }^{3}$ Une voyelle inaccentuée prononcée dans des contextes où la prononciation prescriptive n'indique aucune voyelle finale, par exemple dans [bõzuвə] pour bonjour.
} 
(2004) examine des moments où la clôture d'une séquence est pertinente, et montre qu'une augmentation nette et soudaine de la hauteur mélodique et de la sonie signale que le tour amorce une nouvelle séquence, alors que les expansions de la séquence en cours n'exhibent pas ces caractéristiques prosodiques. Ainsi, à des moments de clôture possible de la séquence, le formatage prosodique fournit un indice de la position séquentielle du tour (réaction ou initiation) - et, réflexivement, la précise. De même, la prosodie des plaintes joue un rôle central pour leur organisation séquentielle : les plaintes concernant un tiers peuvent prendre deux formats (soit registre mélodique élevé, gamme tonale large et sonie forte, soit registre mélodique bas, gamme tonale compressée et sonie moins forte), lesquels correspondent aux deux positions séquentielles où apparaissent les plaintes (Ogden 2010). Alors que l'action de se plaindre, en soi, n'a pas de manifestation prosodique spécifique, les plaintes qui forment une clôture possible de la séquence sont prosodiquement distinguées des plaintes qui cherchent l'affiliation et proposent la continuation de la séquence de plainte.

Bien des travaux sur le rôle de la prosodie dans l'organisation des séquences ont été articulés en termes d'alignement et d'affiliation. Une observation générale est que les études sur ces thèmes engendrent souvent des résultats concernant des manifestations prosodiques relatives. Il ne s'agit donc pas de choix paradigmatiques entre options dans un système, mais de contrastes et similarités prosodiques syntagmatiques entre tours de parole.

Prenons d'abord l'exemple d'un paramètre prosodique unique, le rythme. En anglais (mais plus rarement en italien), les transitions non problématiques sont caractérisées par l'intégration rythmique d'un tour au prochain (la continuation, par-delà de la transition, d'un rythme régulier des syllabes accentuées) (Auer \& al. 1999). Ensuite, l'organisation prosodique et phonétique des paires d'évaluations est aussi relative, mais implique plusieurs paramètres phonétiques : suite à une première évaluation, les évaluations affiliatives sont phonétiquement formatées comme 'amplifiées' (upgraded) par rapport à la première évaluation (avec une augmentation du niveau mélodique, un élargissement de la gamme tonale, une décélération et des articulations plus tendues) (Ogden 2006). Finalement, Szczepek Reed (2006) décrit tout un ensemble de procédés d'orientation prosodique, notamment le recyclage (matching) d'une caractéristique prosodique du tour précédent (indépendamment d'un éventuel recyclage verbal), qu'il s'agisse de son contour intonatif, son registre mélodique (relativement à la tessiture du locuteur), son débit d'articulation, sa sonie globale ou sa qualité de voix. Ces formes de recyclage d'un ou plusieurs traits prosodiques accompagnent de nombreuses actions alignées en deuxième position, mais l'orientation prosodique peut également être employée stratégiquement avec un tour non-aligné, satisfaisant ainsi en apparence des normes d'alignement tout en poursuivant d'autres objectifs interactionnels (Szczepek Reed 2006).

Si les études sur l'interface, en français, entre prosodie et organisation des séquences font largement défaut, nous proposons ici quelques observations préliminaires (issues d'un projet de recherche en cours), relatives à la prosodie de la particule voilà dans son usage comme marqueur de confirmation ou d'accord. Cet usage de voilà est mentionné par Bert et al. (2008), qui notent qu'il a typiquement une intonation descendante (or nous verrons que ce n'est pas toujours le cas). Bert et al. (2008) constatent aussi la récurrence de cette particule par exemple dans les confirmations de complétions collaboratives. Lorsque voilà sert à confirmer, cette particule signale justement l'accès épistémique partagé des locuteurs, alors que le fait même d'être celui qui confirme permet de revendiquer l'autorité épistémique définitive. Ce voilà confirmateur se distingue ainsi par exemple de la particule oui, qui sert à confirmer sans cette reconnaissance du caractère prévisible de ce qui est à confirmer (Oloff 2016). Ainsi s'explique l'usage avec les complétions collaboratives et d'autres confirmables où le destinataire de la confirmation a néanmoins 'un certain degré de savoir' (Oloff op. cit., 244), y compris les formulations (Persson 2013, 30). 
Dans l'extrait (7), nous trouvons deux voilà, formellement distingués par leurs contours intonatifs, et respectivement représentatifs de deux environnements séquentiels distincts bien que voilà serve à confirmer dans les deux cas. Ici, le traiteur TRA vient de noter les coordonnées d'une cliente prospective (CLI), alors que les denrées à livrer n'ont pas encore été détaillées.

Extrait (7) [CORAL.FTELPV17.2:26]
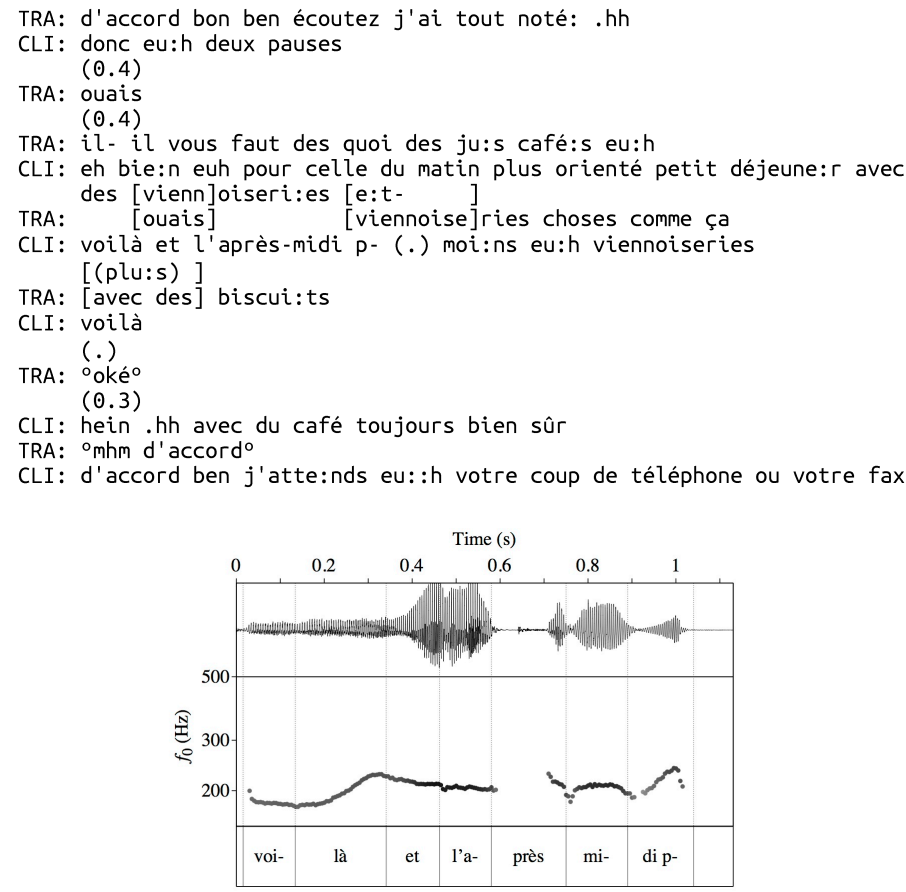

Figure 7a. Oscillogramme et tracé de $f_{0} d u$ fragment voilà et l'après-midi p- (extrait 7 , ligne 10).

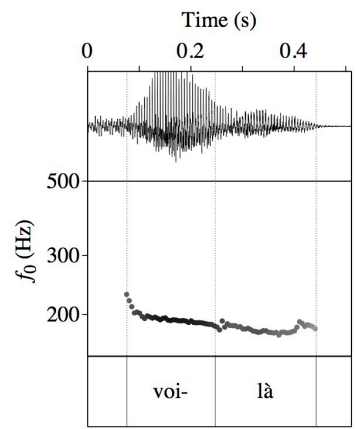

Figure 7b. Oscillogramme et tracé de $f_{0}$ du tour voilà (extrait 7 , ligne 13 ). 
À la ligne 9, TRA énonce une inférence tirée du tour précédent et de la ligne 7 en particulier (étant donné que TRA commence en chevauchement avec la ligne 8). En réponse (ligne 10), un voilà avec une montée intonative (fig. 7a) est produit par CLI. Vu que c'est TRA qui pose la question à la ligne 6 , et vu le statut de cliente prospective de CLI, CLI semble avoir une autorité épistémique ultime sur la commande, et le voilà confirme donc la contribution de TRA (ligne 9) en le traitant comme découlant de ce que CLI elle-même vient de dire. Ainsi, le contour intonatif montant ne semble pas influencer l'action accomplie (qui reste une confirmation) mais plutôt communiquer une certaine analyse séquentielle: la montée intonative indique l'identification d'un projet conversationnel en cours qui n'est pas encore complet. Notamment, il semble que de manière récurrente, le voilà confirmateur avec cette prosodie marque l'achèvement d'une phase actuelle (alors que de subséquentes phases ont été projetées), ainsi projetant le passage à la phase suivante. En effet, ici, lorsque les besoins spécifiques pour la première des deux pauses sont précisés aux lignes 7-8, des précisions relatives à la seconde pause sont également projetées, et CLI enchaîne avec ces précisions directement après son voilà (à noter aussi, la différence d'amplitude qui délimite ce voilà de ce qui suit (fig. 7a). Ensuite, TRA produit à nouveau une inférence tirée du tour de CLI, formatée comme un syntagme prépositionnel (ligne 12, avec des biscui:ts), et CLI confirme encore avec un voilà (ligne 13, fig. $7 \mathrm{~b}$ ). Si le voilà produit à la ligne 13 accomplit aussi une confirmation définitive d'un fait prévisible, en revanche l'intonation descendante implique plutôt la clôture de la séquence actuelle (Mondada à paraître), sans signaler que cette clôture entraîne un passage à quelque chose de déjà projeté (la mention du café à la ligne 17 est présentée comme un ajout d'un fait évident : bien sûr).

Par la prosodie de ce marqueur de confirmation, son émetteur révèle donc une certaine analyse de la trajectoire séquentielle dont le marqueur fait partie, et contribue aussi réflexivement à la réalisation de cette trajectoire. En l'occurrence, cette analyse séquentielle est similaire à celle que propose un continuateur (Schegloff 1982) : une unité plus large est en cours, et pas encore complète. Seulement, ici, cette analyse s'exprime dans une autre position séquentielle : le tour n'est pas produit par le participant qui enregistre et accuse réception, mais par le participant qui est 'l'auteur' de la séquence. Précisons que cette distinction intonative opère indépendamment $\mathrm{du}$ fait que l'émetteur du voilà continue immédiatement son tour (ou non); dans d'autres cas observés, c'est l'autre locuteur qui continue, et entame la phase suivante, directement après le voilà. Donc, ces observations permettent, nous l'espérons, d'entrevoir le travail que la prosodie peut faire non seulement pour la gestion de la parole, mais spécifiquement pour l'organisation des séquences.

\section{Prosodie et construction des actions}

La dernière tâche générale à laquelle contribue la prosodie est la production des actions mêmes (Levinson 2013). Un résultat qui émerge des études qui seront abordées ci-dessous est que la prosodie est une composante dans les mécanismes de construction d'actions - la prosodie ne définit jamais toute seule une pratique conversationnelle. Ainsi, la prosodie peut être analysée comme faisant une contribution - certes importante, parfois même cruciale - au travail interactionnel du tour de parole. Local (2005) montre pour l'anglais que la pratique de proposer une complétion collaborative - fait généralement conçu surtout en termes syntaxiques - a également une composante prosodique : un certain formatage prosodique fait partie intégrante de l'organisation normative des complétions collaboratives, voire permet l'identification d'un tour en tant que complétion collaborative. Dans d'autres cas, des formes intonatives caractéristiques apparaissent avec essentiellement la même fonction dans des actions diverses: Ogden et al. (2004) montrent qu'il y a un contour intonatif montantdescendant en finnois qui présente le contenu du tour même comme étant prévisible, n'ayant 
'rien de neuf'. Ce contour accompagne des actions assez différentes (mais néanmoins restreintes du fait du sens qu'il permet de communiquer), et le travail interactionnel de ce contour se superpose donc à celui par ailleurs effectué par le tour, par exemple clôturer une séquence par une expression idiomatique ou une répétition, formuler une paraphrase du discours précédent, ou accomplir une deuxième partie de paire routinière (Ogden \& al. 2004). De manière similaire, l'emphase d'intensification en anglais (avec des caractéristiques phonétiques telles que durées extrêmement longues et articulations renforcées) n'est pas limitée à un type spécifique d'actions, mais peut modifier entre autres les plaintes, les lancements de récits, et les évaluations renforcées (par exemple les évaluations réitérées, lorsqu'une réponse affiliative à la première fait défaut) (Ogden 2012). Cependant, cette pratique (phonétiquement constituée) n'accompagne pas n'importe quelle action; le fait qu'elle rend plus extrêmes les éléments lexicaux affectés au service du travail du tour dans son ensemble, restreint fortement la gamme des actions susceptibles.

Plus souvent, la prosodie a été analysée du point de vue de son apport à des tours avec des formats spécifiques, tels que les initiations de réparation non ciblées (open class) et les interprétations-candidates (Selting 1996 ; Benjamin 2013 ; Rossi 2015), ainsi que les hétérorépétitions (Kelly \& Local 1989, 263-285 ; Couper-Kuhlen 1996 ; Selting 1996 ; de Fornel \& Léon 1997 ; Benjamin \& Walker 2013 ; Persson 2015b ; Rossi 2015). Ainsi, la variable du contenu lexico-grammatical demeure en quelque sorte constante (du moins en ce qui concerne les aspects pertinents pour l'action), ce qui n'est pas une simple astuce méthodologique pour cerner la contribution de la prosodie, mais un reflet du fait que la prosodie ne fait pas toute seule son travail de signification dans l'interaction (cf. supra). Ces analyses citées montrent que la prosodie peut différencier des actions alternatives, et donc indiquer laquelle, parmi des réponses radicalement différentes, est prochainement pertinente (alors que le matériau verbal en lui-même n'aurait pas suffi). Ainsi, par exemple en italien, les contours intonatifs permettent aux participants de distinguer, d'une part, des répétitions qui demandent simplement une confirmation de la bonne compréhension du tour problématique, et d'autre part, des répétitions qui sollicitent une révision ou correction du tour problématique (Rossi 2015). Les contours intonatifs se sont également avérés distinctifs en français - du point de vue de l'action - dans les formulations (Persson 2013 ; cf. section 2.1). La montée sur la pénultième (avec descente sur la syllabe finale) est le contour intonatif des formulations qui sollicitent une simple confirmation de la compréhension formulée, sans plus. Par contre, les formulations à montée finale servent à solliciter à la fois une confirmation et une explication ou élaboration des faits confirmés (Persson 2013).

Dans certains cas, la prosodie se décrit le mieux comme contribuant avec une action secondaire (Levinson 2013) alors que l'action primaire est autrement accomplie. Un exemple en est l'auto-répétition suite à une hétéro-initiation de réparation, c'est-à-dire l'auto-répétition qui offre une solution dans les séquences de réparations (Curl 2005). Ici, un format phonétique amplifié (prosodie plus ample et configurations articulatoires différentes par rapport au tour problématique) se distingue d'un format 'affaibli' (downgraded) (prosodie moins ample ou similaire et configurations articulatoires semblables par rapport au tour problématique). Le format amplifié indique l'à-propos du tour d'origine, et attribue le trouble à l'interlocuteur qui a initié la réparation; le format affaibli indique que le locuteur assume la responsabilité du trouble, et reconnaît le caractère inopportun du tour d'origine. Ainsi, si l'action primaire (l'effectuation de la réparation) est construite par le fait de se répéter (reproduisant ainsi un tour possiblement mal entendu), l'action secondaire transmise par le format phonétique concerne l'attribution de la responsabilité du trouble (et ces actions secondaires n'ont qu'une incidence occasionnelle sur le déroulement séquentiel (Persson 2015a)). 
Dans un projet en cours sur les hétéro-répétitions dans cinq langues différentes, il est démontré pour le français (Persson à paraître) qu'un contour intonatif particulier (une large montée-descente sur la syllabe à accentuation majeure ${ }^{4}$ ) spécifie que la répétition vise d'abord à faire confirmer ce que l'on a entendu ou compris (pour éliminer d'éventuels troubles moins sérieux comme les problèmes d'audibilité), mais ces répétitions font également autre chose : elles projettent une réaction désaffiliative subséquente, au cas où la version entendue s'avère exacte (Persson à paraître). Ainsi, ces répétitions préfigurent un traitement de problèmes plus sérieux (concernant par exemple l'acceptabilité), et elles sont véritablement prédésaffiliatives, ce que d'autres répétitions (qui, par exemple, sollicitent simplement une confirmation) ne sont pas.

Ici, nous souhaitons souligner notamment que le même contour intonatif apparaît également dans d'autres types de tours que les répétitions, avec une fonction apparemment analogue, tant à l'égard du 'sens pragmatique' que de la trajectoire séquentielle. Une illustration de ce contour dans une interprétation-candidate est offerte dans l'extrait (8). Ici, CLI a appelé sa faculté pour des renseignements sur l'affichage des résultats des maîtrises. FAC a affirmé ne pas avoir reçu l'information cherchée de la part de la personne responsable.

Extrait (8) [UBS.102.0:24]
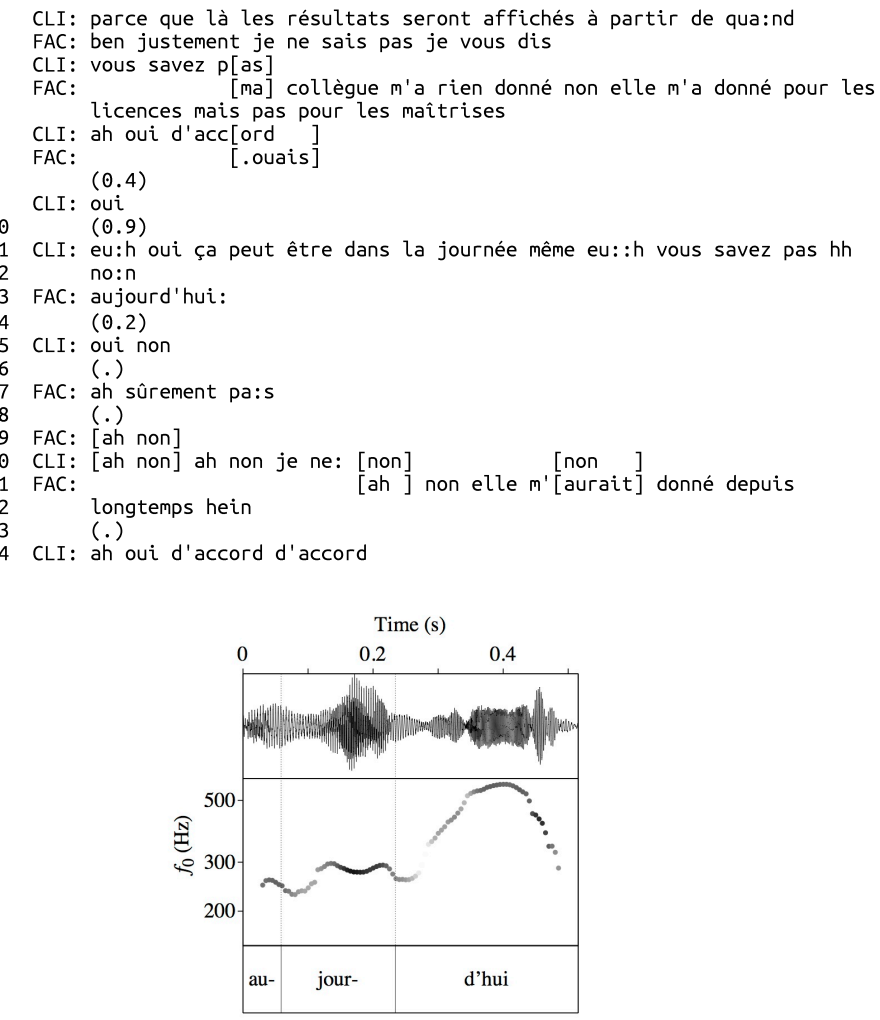

Figure 8. Oscillogramme et tracé de $f_{0}$ du tour aujourd'hui (extrait 8).

\footnotetext{
${ }^{4}$ Parfois appelé intonation d'implication (Portes \& Lancia 2017), ce contour peut être annoté H*L\%.
} 
Lorsque FAC produit aujourd'hui (ligne 13), elle cherche en premier lieu à vérifier son interprétation de la référence temporelle dans la journée même, sollicitant donc une confirmation, mais grâce à l'emploi du contour montant-descendant (fig. 8), FAC préfigure aussi sa propre réaction désaffiliative ultérieure. La réponse de CLI (ligne 15 : oui non), en apparence énigmatique, peut donc être comprise comme un oui qui confirme l'interprétation, suivi d'un non qui anticipe une réponse négative à venir de la part de FAC. En effet, FAC produit une réponse négative vivement exprimée à la ligne 17, réitérée et justifiée dans ce qui suit (lignes 19 et 21-22). Cet extrait montre ainsi une orientation de l'interlocuteur vers les deux aspects du travail interactionnel de ce contour dans cet environnement : la demande de confirmation et le travail pré-désaffiliatif. Le contour joue donc un rôle analogue dans les hétéro-répétitions et dans les interprétations-candidates - deux environnements interactionnels distincts, mais néanmoins apparentés. Nous reviendrons à la question de généralisations possibles dans la section 5 .

Pour conclure, soulignons que les contributions de la prosodie aux actions semblent aussi variées que les actions elles-mêmes. En effet, en tant que composante dans la construction d'actions, la prosodie est réflexivement liée à l'action : la prosodie contribue à constituer l'action, et en même temps la prosodie n'acquiert sa signification que lorsqu'elle est mise à l'œuvre dans un contexte actionnel et séquentiel spécifique.

\section{Conclusions et perspectives}

Dans cet article, nous avons tenté de faire un inventaire des recherches sur le rôle de la prosodie pour l'organisation de l'interaction, en enrichissant cette vue d'ensemble avec des illustrations empiriques focalisées sur la langue française. Notre approche, qui considère successivement trois tâches fondamentales en interaction, présente au moins deux défauts. D'abord, plusieurs pistes actuelles de recherche ne peuvent facilement être résumées en ces termes-là. Pour cette raison (et pour des raisons de limitation d'espace), nous avons ignoré les études sur la prosodie dans l'accomplissement interactionnel d'affectivité (Reber 2012), et dans les interactions avec des groupes spécifiques de participants, comme les enfants (Wells \& Stackhouse 2016) et les personnes ayant des troubles de la parole (Walker \& Local 2013). Ensuite, la séparation des niveaux d'analyse n'est pas totale dans la pratique. Par exemple, le contraste entre montée et montée énumérative analysé dans l'extrait (6) ne concerne pas uniquement la gestion de la parole ; il concerne aussi une différence sur le plan de l'action, même si cette différence ne se traduit pas parfaitement en une paire d'étiquettes (comme par exemple offrir-proposer). Lorsqu'on considère comment les participants se servent de la ressource qu'est la prosodie, l'action, l'organisation des séquences et la gestion de la parole s'avèrent donc étroitement reliées entre elles.

Rappelons les fortes ressemblances observées entre deux usages du contour montantdescendant (voir l'analyse de l'extrait (8)), dans les répétitions et dans les interprétationscandidates. En revanche, le contour avec montée sur la pénultième - qui dans les formulations et les interprétations-candidates sert à solliciter une simple confirmation - ne semble pas avoir cette fonction dans les répétitions (Persson 2013, 36). Au fur et à mesure que le champ d'étude évolue, un défi important du point de vue linguistique sera de découvrir à quel point (et selon quelles modalités) les formes prosodiques et phonétiques, telles les contours intonatifs, ont des fonctions restreintes à un environnement actionnel-séquentiel, et à quel point (et selon quelles modalités) les fonctions ont des 'airs de famille' entre environnements distincts. Avec l'accumulation d'études individuelles, visant des environnements spécifiques, on sera mieux en mesure de comprendre les articulations, et délimitations, des usages situés décrits. 
Ayant considéré la prosodie dans ses interfaces avec trois dimensions centrales de l'organisation interactionnelle, nous concluons en constatant que la prosodie exemplifie parfaitement les détails 'vus mais non remarqués' (Garfinkel 1967, 36) par les participants : un formatage prosodique particulier (et ainsi les multiples inférences que les coparticipants peuvent en tirer) est inévitable lorsqu'on parle. Dès lors, le besoin d'une compréhension analytique des phénomènes prosodiques - quant à la recherche sur l'interaction - découle directement du rôle essentiel de la prosodie pour les participants.

Rasmus Persson

Department of Language and Linguistic Science

University of York

Heslington, York, YO10 5DD, UK

<rasmus.persson@liu.se>

\section{Références}

Auer, P., Couper-Kuhlen, E. \& Müller, F. (1999). Language in Time: The Rhythm and Tempo of Spoken Interaction. Oxford, Oxford University Press.

Barth-Weingarten, D. (2016). Intonation Units Revisited: Cesuras in Talk-in-Interaction. Amsterdam, Benjamins.

Benjamin, T. (2013). Signaling trouble: On the linguistic design of other-initiation of repair in English conversation. Thèse de doctorat, Université de Groningen.

Benjamin, T. \& Walker, T. (2013). Managing problems of acceptability through high rise-fall repetitions. Discourse Processes, 50, 107-138.

Bert, M., Bruxelles, S., Etienne, C. \& al. (2008). Tool-assisted analysis of interactional corpora: voilà in the CLAPI database. Journal of French Language Studies, 18, 121-145.

Bögels, S. \& Torreira, F. (2015). Listeners use intonational phrase boundaries to project turn ends in spoken interaction. Journal of Phonetics, 52, 46-57.

Couper-Kuhlen, E. (1996). The prosody of repetition: On quoting and mimicry. In Couper-Kuhlen, E. \& Selting, M. (eds), Prosody in Conversation, Cambridge, Cambridge University Press, 366-405.

Couper-Kuhlen, E. (2004). Prosody and sequence organization in English conversation. In CouperKuhlen, E. \& Ford, C.E. (eds), Sound Patterns in Interaction, Amsterdam, Benjamins, 335-376.

Curl, T.S. (2005). Practices in other-initiated repair resolution: The phonetic differentiation of 'repetitions'. Discourse Processes, 39, 1-43.

de Fornel, M. \& Léon, J. (1997). Des questions-échos aux réponses-échos: Une approche séquentielle et prosodique des répétitions dans la conversation. Cahiers de praxématique, 28, 101-126.

Delais-Roussarie, É., Post, B., Avanzi, M. \& al. (2015). Intonational phonology of French: Developing a ToBI system for French. In Frota, S. \& Prieto, P. (eds), Intonation in Romance, Oxford, Oxford University Press, 63-100.

Di Cristo, A. (1998). Intonation in French. In Hirst, D. \& Di Cristo, A. (eds), Intonation Systems: A Survey of Twenty Languages, Cambridge, Cambridge University Press, 195-218.

Fagyal, Z. \& Moisset, C. (1999). Sound change and articulatory release: Where and why are high vowels devoiced in Parisian French? In Proceedings of the $14^{\text {th }}$ International Congress of Phonetic Sciences. Vol. 1, San Francisco, 309-312.

Ford, C.E. (2004). Contingency and units in interaction. Discourse Studies, 6, 27-52.

Ford, C.E., Fox, B.A. \& Thompson, S.A. (1996). Practices in the construction of turns: The 'TCU' revisited. Pragmatics, 6, 427-454.

Ford, C.E., Fox, B.A. \& Thompson, S.A. (2013). Units and/or action trajectories? The language of grammatical categories and the language of social action. In Szczepek Reed, B. \& Raymond, G. (eds), Units of Talk-Units of Action, Amsterdam, Benjamins, 13-56. 
Fox, B.A. (2001). An exploration of prosody and turn projection in English conversation. In Selting, M. \& Couper-Kuhlen, E. (eds), Studies in Interactional Linguistics, Amsterdam, Benjamins, 287-315.

French, P. \& Local, J. (1983). Turn-competitive incomings. Journal of Pragmatics, 7, 17-38.

Garfinkel, H. (1967). Studies in Ethnomethodology. Englewood Cliffs, NJ, Prentice-Hall.

Hansen, A.B. \& Mosegaard Hansen, M.B. (2003). Le [ə] prépausal et l'interaction. In Hansen, A.B. \& Mosegaard Hansen, M.B. (éds), Structure linguistiques et interactionnelles dans le français parlé, Copenhague, Museum Tusculanum Press, 89-109.

Heritage, J. (1989). Current developments in conversation analysis. In Bull, P. \& Roger, D. (eds), Conversation: An Interdisciplinary Perspective, Clevedon, Multilingual Matters, 21-47.

Heritage, J. \& Watson, R. (1979). Formulations as conversational objects. In Psathas, G. (ed.), Everyday Language: Studies in Ethnomethodology, New York, Irvington, 123-162.

Jefferson, G. (1990). List-construction as a task and resource. In Psathas, G. (ed.), Interaction Competence, Washington, IIEMCA \& University Press of America, 63-92.

Kelly, J. \& Local, J. (1989). Doing Phonology. Manchester, Manchester University Press.

Levinson, S.C. (2013). Action formation and ascription. In Sidnell, J. \& Stivers, T. (eds), The Handbook of Conversation Analysis, Chichester, Wiley-Blackwell, 103-130.

Local, J. (1992). Continuing and restarting. In Auer, P. \& Di Luzio, A. (eds), The Contextualization of Language, Amsterdam, Benjamins, 273-296.

Local, J. (2005). On the interactional and phonetic design of collaborative completions. In Hardcastle, W.J. \& Mackenzie Beck, J. (eds), A Figure of Speech, Mahwah, NJ, Erlbaum, 263-282.

Local, J. \& Kelly, J. (1986). Projection and 'silences': Notes on phonetic and conversational structure. Human Studies, 9, 185-204.

Local, J., Kelly, J. \& Wells, B. (1986). Towards a phonology of conversation: Turn-taking in Tyneside English. Journal of Linguistics, 22, 411-437.

Local, J. \& Walker, G. (2004). Abrupt-joins as a resource for the production of multi-unit, multi-action turns. Journal of Pragmatics, 36, 1375-1403.

Local, J. \& Walker, G. (2005). Methodological imperatives for investigating the phonetic organization and phonological structures of spontaneous speech. Phonetica, 62, 120-130.

Local, J. \& Walker, G. (2012). How phonetic features project more talk. Journal of the International Phonetic Association, 42, 255-280.

Local, J., Wells, B. \& Sebba, M. (1985). Phonology for conversation: Phonetic aspects of turn delimitation in London Jamaican. Journal of Pragmatics, 9, 309-330.

Mondada, L. (à paraître). Turn-initial voilà: Reaffirming epistemic authority over the sequence. In Heritage, J. \& Sorjonen, M.L. (eds), Turn-initial particles across languages, Amsterdam, Benjamins.

Mondada, L. (2007). L'interprétation online par les co-participants de la structuration du tour in fieri en TCUs: Évidences multimodales. Travaux Neuchâtelois de Linguistique, 47, 7-38.

Nicolas, P., Letellier-Zarshenas, S., Schadle, I. \& al. (2002). Towards a large corpus of spoken dialogue in French that will be freely available. In Proceedings of the $3^{\text {rd }}$ International Conference on Language Resources and Evaluation, Las Palmas, 649-654.

Ogden, R. (2001). Turn transition, creak and glottal stop in Finnish talk-in-interaction. Journal of the International Phonetic Association, 31, 139-152.

Ogden, R. (2004). Non-modal voice quality and turn-taking in Finnish. In Couper-Kuhlen, E. \& Ford, C.E. (eds), Sound Patterns in Interaction, Amsterdam, Benjamins, 29-62.

Ogden, R. (2006). Phonetics and social action in agreements and disagreements. Journal of Pragmatics, $38,1752-1775$.

Ogden, R. (2010). Prosodic constructions in making complaints. In Barth-Weingarten, D., Reber, E., \& Selting, M. (eds), Prosody in Interaction, Amsterdam, Benjamins, 81-103.

Ogden, R. (2012). Making sense of outliers. Phonetica, 69, 48-67.

Ogden, R., Hakulinen, A. \& Tainio, L. (2004). Indexing 'no news' with stylization in Finnish. In CouperKuhlen, E. \& Ford, C.E. (eds), Sound Patterns in Interaction, Amsterdam, Benjamins, 299-334. 
Oloff, F. (2016). Comparaison de l'emploi de deux marqueurs d'affirmation dans des séquences de coconstruction : voilà et genau. Testi e linguaggi, 10, 243-267.

Persson, R. (à paraitre). Prosody and grammar of next-turn repetitions in French. Language in Society.

Persson, R. (2013). Intonation and sequential organization: Formulations in French talk-in-interaction. Journal of Pragmatics, 57, 19-38.

Persson, R. (2014). Ressources linguistiques pour la gestion de l'intersubjectivité dans la parole en interaction. Analyses conversationnelles et phonétiques. Thèse de doctorat, Université de Lund.

Persson, R. (2015a). Indexing one's own previous action as inadequate: On $a h$-prefaced repeats as receipt tokens in French talk-in-interaction. Language in Society, 44, 497-524.

Persson, R. (2015b). Registering and repair-initiating repeats in French talk-in-interaction. Discourse Studies, 17, 583-608.

Persson, R. (2017). Fill-in-the-blank questions in interaction: Incomplete utterances as a resource for doing enquiries. Research on Language and Social Interaction, 50, 227-248.

Pomerantz, A. (1988). Offering a candidate answer: An information seeking strategy. Communication Monographs, 55, 360-373.

Portes, C., Bertrand, R. \& Espesser, R. (2007). Contribution to a grammar of intonation in French: Form and function of three rising patterns. Nouveaux cahiers de linguistique française, 28, 155-162.

Portes, C. \& Lancia, L. (2017). Earlier or higher? Comparing French rising-falling contour with rising contour in a corpus of conversation. Journal of Phonetics, 63, 35-52.

Reber, E. (2012). Affectivity in Interaction: Sound Objects in English. Amsterdam, Benjamins.

Rossi, G. (2015). Other-initiated repair in Italian. Open Linguistics, 1, 256-282.

Sacks, H. (1984). Notes on methodology. In Atkinson, J.M. \& Heritage, J. (eds), Structures of Social Action, Cambridge, Cambridge University Press, 21-27.

Schegloff, E.A. (1982). Discourse as an interactional achievement: Some uses of 'uh huh' and other things that come between sentences. In Tannen, D. (ed.), Analyzing Discourse, Washington, Georgetown University Press, 71-93.

Schegloff, E.A. (1998). Reflections on studying prosody in talk-in-interaction. Language and Speech, 41, 235-263.

Selting, M. (1996). Prosody as an activity-type distinctive cue in conversation: The case of so-called 'astonished' questions in repair initiation. In Couper-Kuhlen, E. \& Selting, M. (eds), Prosody in Conversation, Cambridge, Cambridge University Press, 231-270.

Selting, M. (2000). The construction of units in conversational talk. Language in Society, 29, 477-517.

Selting, M. (2007). Lists as embedded structures and the prosody of list construction as an interactional resource. Journal of Pragmatics, 39, 483-526.

Szczepek Reed, B. (2004). Turn-final intonation in English. In Couper-Kuhlen, E. \& Ford, C.E. (eds), Sound Patterns in Interaction, Amsterdam, Benjamins, 97-118.

Szczepek Reed, B. (2006). Prosodic Orientation in English Conversation. Basingstoke, Palgrave.

Szczepek Reed, B. (2010). Intonation phrases in natural conversation: A participants' category? In BarthWeingarten, D., Reber, E. \& Selting, M. (eds), Prosody in Interaction, Amsterdam, Benjamins, 191212.

Torreira, F. \& Valtersson, E. (2015). Phonetic and visual cues to questionhood in French conversation. Phonetica, 72, 20-42.

Walker, G. (2004). On some interactional and phonetic properties of increments to turns in talk-ininteraction. In Couper-Kuhlen, E. \& Ford, C.E. (eds), Sound Patterns in Interaction, Amsterdam, Benjamins, 147-169.

Walker, G. (2010). The phonetic constitution of a turn-holding practice: Rush-throughs in English talk-ininteraction. In Barth-Weingarten, D., Reber, E. \& Selting, M. (eds), Prosody in Interaction, Amsterdam, Benjamins, 51-72.

Walker, G. (2012). Coordination and interpretation of vocal and visible resources: 'Trail-off' conjunctions. Language and Speech, 55, 141-163.

Walker, G. (2013). Phonetics and prosody in conversation. In Sidnell, J. \& Stivers, T. (eds), The Handbook of Conversation Analysis, Chichester, Wiley-Blackwell, 455-474. 
Walker, G. (2017). Pitch and the projection of more talk. Research on Language and Social Interaction, 50, 206-225.

Walker, G. \& Local, J. (2013). On the intersection of phonetic detail and the organization of interaction: Clinical connections. Clinical Linguistics \& Phonetics, 27, 770-783.

Wells, B. \& MacFarlane, S. (1998). Prosody as an interactional resource: Turn-projection and overlap. Language and Speech, 41, 265-294.

Wells, B. \& Peppé, S. (1996). Ending up in Ulster: Prosody and turn-taking in English dialects. In Couper-Kuhlen, E. \& Selting, M. (eds), Prosody in Conversation, Cambridge, Cambridge University Press, 101-130.

Wells, B. \& Stackhouse, J. (2016). Children's Intonation: A Framework for Practice and Research. Chichester, Wiley-Blackwell. 